\title{
Evaluation of Lethal Giant Larvae as a Schistosomiasis Vaccine Candidate
}

\author{
Yufan Cao, ${ }^{1}$ Hongbin Qiao, ${ }^{1}$ Yanli Shi, ${ }^{1}$ Yu Han, ${ }^{1}$ Jinming Liu, ${ }^{1}$ Hao Li, ${ }^{1}$ \\ Ke Lu, ${ }^{1}$ Jiaojiao Lin, ${ }^{1,2}$ and Yamei Jin ${ }^{1}$ \\ ${ }^{1}$ Shanghai Veterinary Research Institute, Chinese Academy of Agricultural Sciences, Key Laboratory of Animal Parasitology, \\ Ministry of Agriculture, Shanghai 200241, China \\ ${ }^{2}$ Jiangsu Coinnovation Center for Prevention and Control of Important Animal Infectious Diseases and Zoonoses, Yangzhou, \\ Jiangsu Province 225009, China \\ Correspondence should be addressed to Yamei Jin; yameijin@shvri.ac.cn
}

Received 19 April 2016; Revised 20 July 2016; Accepted 11 August 2016

Academic Editor: David Piedrafita

Copyright (C) 2016 Yufan Cao et al. This is an open access article distributed under the Creative Commons Attribution License, which permits unrestricted use, distribution, and reproduction in any medium, provided the original work is properly cited.

\begin{abstract}
Schistosomiasis is a neglected tropical disease of humans, and it is considered to be the second most devastating parasitic disease after malaria. Eggs produced by normally developed female worms are important in the transmission of the parasite, and they responsible for the pathogenesis of schistosomiasis. The tumor suppressor gene lethal giant larvae (lgl) has an essential function in establishing apical-basal cell polarity, cell proliferation, differentiation, and tissue organization. In our earlier study, downregulation of the lgl gene induced a significant reduction in the egg hatching rate of Schistosoma japonicum (Sj) eggs. In this study, the Sjlgl gene was used as a vaccine candidate against schistosomiasis, and vaccination achieved and maintained a stable reduction of the egg hatching rate, which is consistent with previous studies, in addition to reducing the worm burden and liver egg burden in some trials.
\end{abstract}

\section{Introduction}

Schistosomiasis (an infection caused by the blood fluke schistosomes) is the second most common, after malaria, parasitic disease of human and animals worldwide, and it occurs mainly in developing countries. Despite decades of control, there are still millions of people at risk of contracting this infection [1]. Most current schistosomiasis control strategies are based on the use of safe and effective drugs, such as praziquantel and oxamniquine, but these do not prevent reinfection, and the number of infected people has remained constant [2]. The best long-term strategy for the control of schistosomiasis is through immunization with a schistosomiasis vaccine in combination with drug treatment [3]. To date, candidate antigens suggested by the World Health Organization and reported by researchers are not sufficiently protective to be used in the clinic [4]; therefore, it is necessary to search for new, highly protective vaccine candidates.
Lethal giant larvae (LGL) is a member of the SCRIB complex, which interact to regulate the polarity of cells. LGL is a critical molecular integrator of apical and basolateral activities. The WD40 repeats of LGL form $\beta$-propellers that can act as protein-interacting modules for SCRIB, and the atypical protein kinase C- (aPKC-) mediated phosphorylation of LGL during epithelial polarity establishment is needed for its basolateral localization [5-9]. Many studies have shown that LGL function is essential for the development of polarized epithelia [7, 10-12], localization of cell-fate determinants, and association with the cytoskeletal complex $[13,14]$. LGL was first described in the fruit fly Drosophila melanogaster as a neoplastic tumor suppressor gene $[15,16]$. The tumorous phenotype of the fruit fly is a giant larva [8].

Evolutionarily conserved homologs of $l g l$ have been identified in many species, including human, mouse, and worm [17-19] and, in our earlier study, we found that downregulation of the Sjlgl gene affects not only egg hatching but also parasite morphology and that SjLGL mostly localizes to the 
tegument of adult $S$. japonicum, even though SjLGL does not contain a signal peptide or transmembrane domain [20]. In this study, we evaluated SjLGL as a candidate schistosomiasis vaccine. The results showed that specific antibodies were induced following immunization with recombinant SjLGL (rSjLGL), and there were reductions of the egg hatching rate, worm burden, and liver egg burden, although these were not observed in every trial.

\section{Materials and Methods}

2.1. Molecular Characterization of Sjlgl. The $5333 \mathrm{bp}$ Sjlgl sequence was submitted to GenBank (accession number KF246684) and the selected amino acid sequence in this study was translated from bp 1866-2711. The sequence was analyzed as follows. Signal peptide prediction was performed with the SignalP 3.0 server (http://www.cbs.dtu.dk/services/ SignalP/). Transmembrane helices were analyzed using the TMHMM server version 2.0 (http://www.cbs.dtu.dk/services/ TMHMM-2.0/). The molecular weight (MW) and isoelectric point $(\mathrm{pI})$ were calculated using the ExPASy compute tool (http://www.expasy.ch/tools/pitool.html). The amino acid sequences of the LGL protein were obtained from GenBank and aligned using ClustalX software (http://www.clustal .org/).

2.2. Sjlgl Cloning and Protein Expression. Upstream and downstream oligonucleotides, $5^{\prime}$-CCGGAATTCATAGTCGCTCTAGGCCATTC- $3^{\prime}$ and $5^{\prime}$-CCCAAGCTTTCAGTTAAACTTCTTTCGGTG-3' (EcoRI and HindIII sites are underlined, resp.), were used to amplify the partial Sjlgl coding sequence (GenBank accession number AY812588) from bp 1866-2711. The $846 \mathrm{bp}$ fragment was generated by polymerase chain reaction (PCR) and cloned into the pET28a (+) vector (Novagen, Darmstadt, Germany), and its identity was confirmed by sequencing.

Overexpression of rSjLGL, with an aminoterminal histidine tag, in Escherichia coli BL21 (DE3) cells (Tiangen Biotech Co., Ltd., Beijing, China) was induced by treatment with $1 \mathrm{mM}$ isopropyl- $\beta$-D-thiogalactopyranoside (IPTG) at $37^{\circ} \mathrm{C}$ for $5 \mathrm{~h}$. Bacteria were harvested by centrifugation at $10,000 \times \mathrm{g}$ for $15 \mathrm{~min}$. The supernatant was discarded, and the pellet was suspended in $50 \mathrm{~mL}$ of phosphate-buffered saline (PBS, $\mathrm{pH}$ 7.4) and lysed using ultrasound to release the fusion protein. Following centrifugation at $10,000 \times \mathrm{g}$ for $15 \mathrm{~min}$, the inclusion body protein was extracted in PBS containing urea and purified by passage through Ni-NTA His-Bind Resin (Qiagen GmbH, Hilden, Germany).

2.3. Immunization of Mice. Six-week-old male BALB/c mice were divided into two groups of 10 mice each. Animals in the experimental group were injected subcutaneously with $50 \mu \mathrm{g}$ of rSjLGL fusion protein on $\mathrm{d} 0,15$, and 30 . The recombinant protein was formulated with Montanide ISA 206 (Guoyao, Shanghai, China) at a ratio of $46: 54$. Animals in the control group were administered adjuvant in PBS using the same immunization protocol. The experiments were repeated five times.
Animal care and experimental procedures were conducted in accordance with the guidelines of the Shanghai Veterinary Research Institute for the Care and Use of Laboratory Animals.

2.4. Challenge Infection and Worm Burden Recovery. Two weeks after the last boost, mice were infected via exposure of abdominal skin for at least $15 \mathrm{~min}$ to water containing 40 \pm 5 cercariae. At $42 \mathrm{~d}$ after infection, mice were sacrificed to recover parasites by perfusion via the hepatic vein, and their livers were collected. The worm burden reduction was calculated as [21]

$$
\mathrm{PL}=\left(1-\frac{\mathrm{WREG}}{\mathrm{WRCG}}\right) \times 100 \%,
$$

where PL is the protection level; WRCG is the number of worms recovered from the control group; and WREG is the number of worms recovered from the experimental group.

2.5. Liver Egg Count and Miracidium Hatching Rate. To evaluate the liver egg burden, the liver was weighed and homogenized, and $1 \mathrm{~mL}$ of homogenate was mixed with $1 \mathrm{~mL}$ of $10 \%(\mathrm{w} / \mathrm{v}) \mathrm{NaOH}$ and heated for $10-15 \mathrm{~min}$ at $56^{\circ} \mathrm{C}$. The eggs were counted, and the number of eggs/g was compared to the control mice, using the following formula:

$$
\mathrm{PL}=\left(1-\frac{\mathrm{EPGEG}}{\mathrm{EPGCG}}\right) \times 100 \%,
$$

where EPGCG is the number of eggs/g of the control group and EPGEG is the number of eggs/g of the experimental group.

To hatch the miracidia, $4 \mathrm{~mL}$ of liver homogenate was added to a flask filled with chlorine-free water. The neck of the flask was filled with a very thin layer of absorbent cotton (avoiding air bubbles, which could obstruct the miracidia) and kept at $27^{\circ} \mathrm{C}$ in the light. The supernatant above the cotton, which included the miracidia, was collected at $2 \mathrm{~h}$ and at $4 \mathrm{~h}$ after hatching. The miracidia were fixed with iodine and collected by centrifuging the supernatant at $4000 \times \mathrm{g}$ for $5 \mathrm{~min}$ at $4^{\circ} \mathrm{C}$ and counted under a light microscope (magnification $40 \mathrm{x}$ ). The average egg hatching rate was calculated as miracidium/added egg, and the reduction in the hatching rate was calculated by comparing the results to the control group:

$$
\mathrm{PL}=\left(1-\frac{\mathrm{HREG}}{\mathrm{HRCG}}\right) \times 100 \%
$$

where HRCG is the egg hatching rate of the control group and HREG is the egg hatching rate of the experimental group.

2.6. Measurement of Anti-SjLGL Antibodies. Following immunization, sera were collected at $0,15,30$, and $45 \mathrm{~d}$ after the first immunization ( 2 weeks after each immunization) for measurement of IgG, and sera were collected at 0,10 , 25 , and $40 \mathrm{~d}$ after the first immunization (10 d after each immunization) for measurement of IgE. Specific anti-SjLGL antibodies were measured using an indirect enzyme-linked 


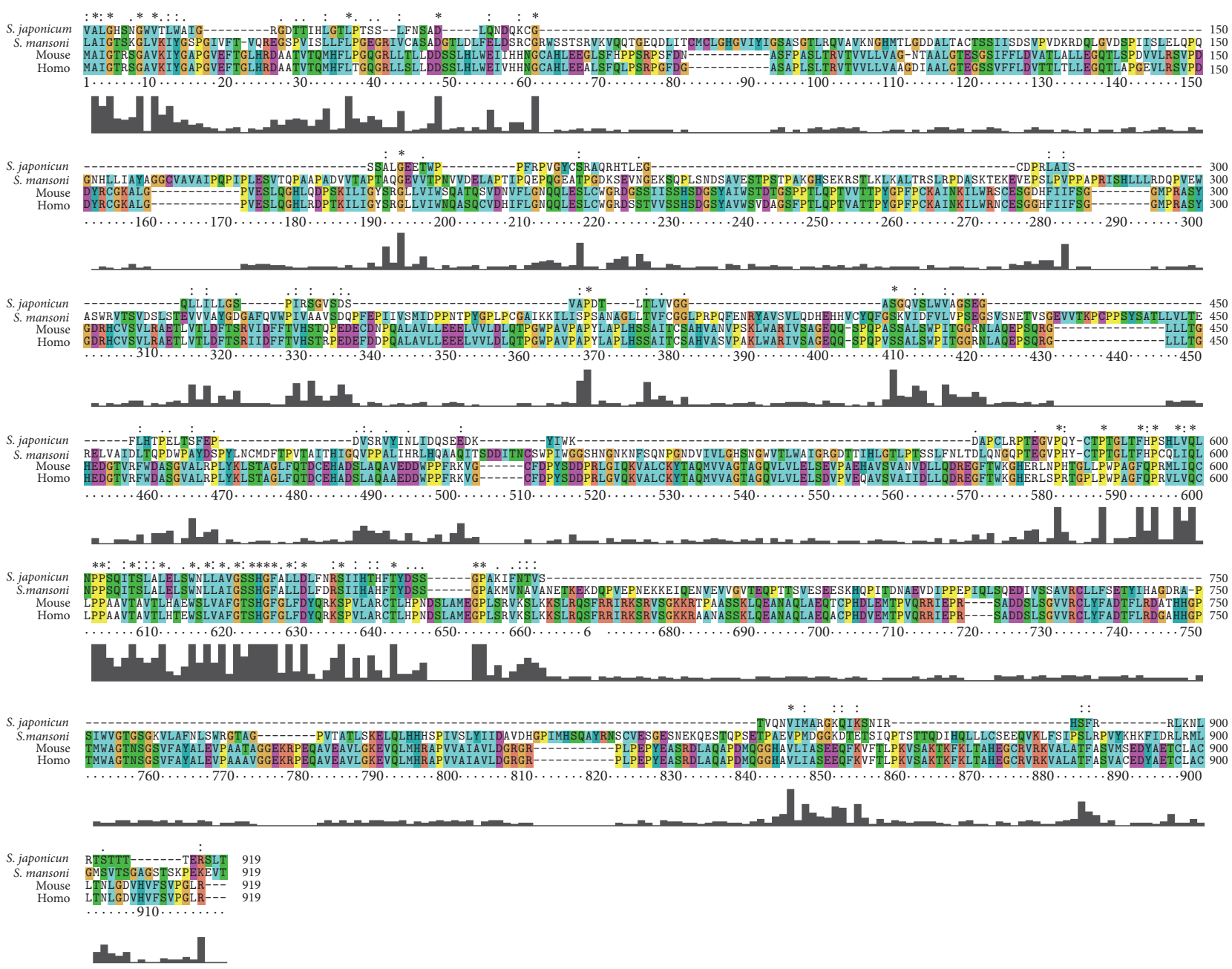

FIGURE 1: The selected protein sequence of SjLGL in relation to S. mansoni, M. musculus, and H. sapiens LGL sequences. ClustalX alignment of the derived amino acid sequences of SmLGL (XP_002577079.1), MmLGL (NP_001152876), and HsLGL (NP_004131.3). Regions with a high level of identity and similarity between the LGL sequences are shown in color.

immunosorbent assay (ELISA). First, 96-well microtiter plates were coated with $100 \mu \mathrm{L}$ of $10 \mu \mathrm{g} / \mathrm{mL}$ rSjLGL overnight at $4^{\circ} \mathrm{C}$ and then blocked for $2 \mathrm{~h}$ at room temperature with $150 \mu \mathrm{L} /$ well of PBS containing $1.5 \%(\mathrm{w} / \mathrm{v})$ bovine serum albumin. A $100 \mu \mathrm{L}$ volume of each serum sample was diluted $1: 100(\mathrm{v} / \mathrm{v})$ in PBS, added to each well, and incubated for $1 \mathrm{~h}$ at room temperature. Plate-bound antibody was detected using peroxidase-conjugated anti-mouse IgG (Dingguo, Shanghai, China) or anti-mouse IgE (AbD Serotec, Raleigh, NC, USA) diluted in PBS 1:5,000 (v/v) or 1:1,000 (v/v), respectively.

2.7. Statistical Analysis. Data are expressed as mean \pm standard deviation (SD). Statistical analysis was done with Student's $t$-test or one-way analysis of variance. The level of a statistically significant difference was set at $p<0.05$.

\section{Results}

3.1. Sequence Analysis of Sjlgl. The posttranslational modifi-

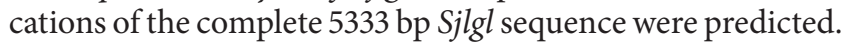

The results showed that Sjlgl encodes a protein of 1435 amino acid residues, with a predicted molecular mass of $\sim 155.68 \mathrm{kDa}$ and a $\mathrm{pI}$ of 5.02; the protein does not contain a signal peptide or transmembrane helix.

The 849 bp sequence of the selected Sjlgl segment encodes a protein of 282 amino acid residues, with a predicted molecular mass of $\sim 30.76 \mathrm{kDa}$ and a $\mathrm{pI}$ of 6.85 . A comparison of the amino acid sequences showed that the LGL segment of $S$. japonicum that was selected in our experiment shared $86 \%, 36 \%$, and 33\% identity with its orthologs in Schistosoma mansoni, Mus musculus, and Homo sapiens, respectively (Figure 1).

3.2. Production of $r S j L G L$. The partial sequence from bp 1866-2711 of Sjlgl was obtained by PCR amplification, cloned into the pET28 expression vector, and expressed in E. coli BL21 (DE3) cells via IPTG induction. During sodium dodecyl sulfate-polyacrylamide gel electrophoresis (SDS-PAGE) $(12 \%(\mathrm{w} / \mathrm{v})$ polyacrylamide), the histidine-tagged protein ran as a single band with a MW of $\sim 36 \mathrm{kDa}$ (Figure 2), which is 


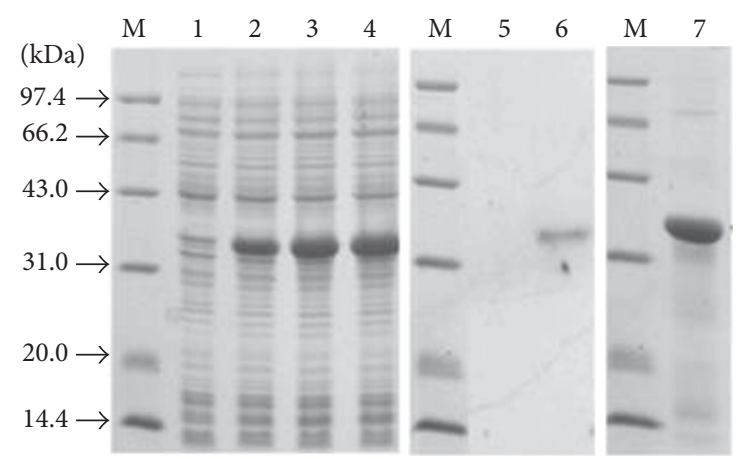

FIgURe 2: Expression and purification of rSjLGL in E. coli. Cell extracts and fractions from E. coli BL21 (DE3) cells that were transformed with the pET28-Sjlgl vector were separated by $12 \%$ SDS-PAGE. Lane M: size markers. Lane 1: total extract of a clone before induction. Lanes 2, 3, and 4: total extracts of a clone 2, 4, and $6 \mathrm{~h}$ after induction with $1 \mathrm{mM}$ IPTG. Lanes 5 and 6: western blot using a polyclonal anti-rSjLGL antibody (lane 5: antigen (total extract of a clone before IPTG induction); lane 6: total extract of a clone after $5 \mathrm{~h}$ of IPTG induction; lane 7: protein purified from the supernatant by passage through Ni-NTA His-Bind Resin).

in accordance with the predicted MW of the rSjLGL protein. Bacteria were lysed using ultrasound, and the lysate was separated into soluble and insoluble fractions; the insoluble fraction contained the majority of the recombinant protein. Next, the soluble protein was purified by passage through Ni-NTA His-Bind resin, and the main recombinant protein in the eluted fractions was pooled (Figure 2, lane 7). After purification, the concentration of protein was $\sim 5 \mathrm{mg} / \mathrm{mL}$, and this was used to immunize mice.

3.3. Evaluation of Anti-SjLGL IgG and IgE Antibodies. To evaluate the presence of anti-SjLGL IgG and IgE antibodies, sera from 10 vaccinated mice from each group were tested using an indirect ELISA. Significant titers of specific antiSjLGL IgG antibodies were detected at all time points tested after the first immunization. The results of the protein immunization experiment showed that the IgG level increased to a high level after the second immunization and remained at that level (Figure 3(a)). The IgE level increased slowly after the first immunization, and it was significantly higher than that of the PBS control group (Figure 3(b)).

3.4. SjLGL Immunization Induces a Protective Effect. To measure the protection levels induced by SjLGL immunization, mice were challenged with $40 \pm 5$ cercariae, and reductions of the worm burden, liver egg burden, and egg hatching rate were determined (Table 1).

Protein vaccination was repeated five times, and, following immunization, worm burden and liver egg burden reductions were observed in two and three, respectively, of the five experimental tests; while the worm burden and liver egg burden reductions did not significantly differ from those of the control group in the other tests, the egg hatching rate reductions were quite similar in these tests, and they reached $75.84 \%$ (Table 1).

\section{Discussion}

Schistosomiasis continues to be a significant global public health concern, and an effective control strategy will require the development of effective vaccines. In recent reports, the use of recombinant antigens, for example, Sm-TSP-2 and Sm 29 induced $\sim 50 \%$ reductions of the worm burden, thereby demonstrating that a vaccine against schistosomes is achievable [21, 22], which led us to search for additional vaccine candidates.

The lethal giant larvae ( $\mathrm{gl} l$ ) gene is well conserved among species, and it functions to link apical-basal cell polarity regulation to cell proliferation control in epithelial tissues $[11,23]$. Mutation of $l g l$ can cause cellular overproliferation, leading to tissue overgrowth (tumors). Mutation of $l g l$ in $D$. melanogaster results in the loss of apical-basal polarity and uncontrolled proliferation, which results in neoplastic tumors $[11,24,25]$. The $l g l$ gene has a remarkable impact on the larval stage in flies and it makes large maternal contributions. Homozygous $l g l$ mutant embryos from a heterozygous mother will progress into the larval stage and then die because of polarity defects when the $l g l$ products from the mother are exhausted. Based on our RNA interference experiments, we think LGL plays important roles in the development of S. japonicum embryos [20]. Additionally, we found that the partial sequence from 1866-2711 bp of Sjlgl is conserved in mammalian $l g l$ genes; thus, its potential as a vaccine candidate was investigated. In this study, immunization with SjLGL achieved a stable and significant reduction of the egg hatching rate; the reduction of the egg hatching rate was $75.84 \%$, and it remained stable at $>50 \%$. The effect of SjLGL immunization on the egg hatching rate might be related to the function of LGL in cell polarity regulation. We did not observe a statistically significant reduction of the worm burden or the liver egg burden in each trial. We believe that differences among individual mice were related to differences in the number and the male/female ratio of cercariae, although the reduction of the egg hatching rate was statistically significant and stable. We tested the combined DNA plus protein vaccination strategy in this study because a similar strategy yielded good results in an HIV vaccine study [26]; however, the combined vaccination strategy did not exhibit greater protective efficacy than a single-protein vaccination strategy (data not shown). This may be because viruses and parasites induce different immune responses.

A mature female $S$. japonicum discharges 5003500 eggs/d [27], which is important for pathological changes in the host liver and, together with the egg hatching rate, is responsible for spreading schistosomiasis. Immature eggs that are newly deposited in the host liver by female worms do not result in granuloma formation, and they do not induce serious pathological changes. The eggs mature after 10-11 d of development; the miracidium in mature eggs will secrete some soluble egg antigen, which causes the granulomatous lesions and hepatomegaly. In the present study, immunization with SjLGL resulted in a statistically significant and stable reduction of the egg hatching rate. In our earlier study, vaccinating mice with SjLGL generated a Thl-type immune response, which protects against 
TABLE 1: Protection level induced in BALB/c mice following immunization with rSjLGL and infection with 40 cercariae.

\begin{tabular}{|c|c|c|c|}
\hline & $\begin{array}{c}\text { Adult worms } \\
\text { mean } \pm \text { SD } \\
(\% \text { reduction })\end{array}$ & $\begin{array}{c}\text { Liver eggs } \\
\text { mean } \pm \text { SD } \\
(\% \text { reduction })\end{array}$ & Egg hatching rate (\% reduction) \\
\hline \multicolumn{4}{|l|}{ Test 1} \\
\hline PBS+ISA206 & $12 \pm 5.78$ & $15446.27 \pm 4518.95$ & $6.43 \pm 2.44$ \\
\hline \multirow[t]{2}{*}{ rSjLGL+ISA206 } & $12.6 \pm 6.22$ & $17286.02 \pm 5933.09$ & $1.55 \pm 0.79$ \\
\hline & $(-7.57)$ & $(-11.91)$ & $\left(75.84^{* *}\right)$ \\
\hline \multicolumn{4}{|l|}{ Test 2} \\
\hline PBS+ISA206 & $14.5 \pm 5.19$ & $60374.84 \pm 26931.24$ & $2.42 \pm 0.96$ \\
\hline \multirow[t]{2}{*}{ rSjLGL+ISA206 } & $18.2 \pm 2.94$ & $64699.45 \pm 32697.79$ & $1.09 \pm 0.29$ \\
\hline & $(-25.51)$ & $(-7.16)$ & $\left(54.62^{* *}\right)$ \\
\hline \multicolumn{4}{|l|}{ Test 3} \\
\hline PBS+ISA206 & $13.9 \pm 5.68$ & $35537.82 \pm 17296.66$ & $4.20 \pm 2.13$ \\
\hline \multirow[t]{2}{*}{ rSjLGL+ISA206 } & $12.66 \pm 5.14$ & $17820.01 \pm 8997.366$ & $1.96 \pm 0.90$ \\
\hline & $(8.87)$ & $\left(49.86^{* *}\right)$ & $\left(53.24^{* *}\right)$ \\
\hline \multicolumn{4}{|l|}{ Test 4} \\
\hline PBS+ISA206 & $22 \pm 8.07$ & $30513.28 \pm 13690.01$ & $4.15 \pm 2.6$ \\
\hline \multirow[t]{2}{*}{ rSjLGL+ISA206 } & $11 \pm 2.7$ & $25081.94 \pm 15991.31$ & $1.59 \pm 1.01$ \\
\hline & $\left(50.0^{*}\right)$ & $\left(33.47^{*}\right)$ & $\left(61.71^{*}\right)$ \\
\hline \multicolumn{4}{|l|}{ Test 5} \\
\hline PBS+ISA206 & $17.14 \pm 3.76$ & $50135.58 \pm 7204.55$ & $7.57 \pm 3.43$ \\
\hline \multirow[t]{2}{*}{ rSjLGL+ISA206 } & $14.92 \pm 6.19$ & $35478.2 \pm 9444.48$ & $3.44 \pm 1.19$ \\
\hline & $\left(12.91^{*}\right)$ & $\left(29.23^{*}\right)$ & $\left(54.49^{*}\right)$ \\
\hline
\end{tabular}

Tests $1-5, n=10 .{ }^{*}$ Statistically significant compared with the control group $(p<0.05),{ }^{* *}(p<0.01)$.

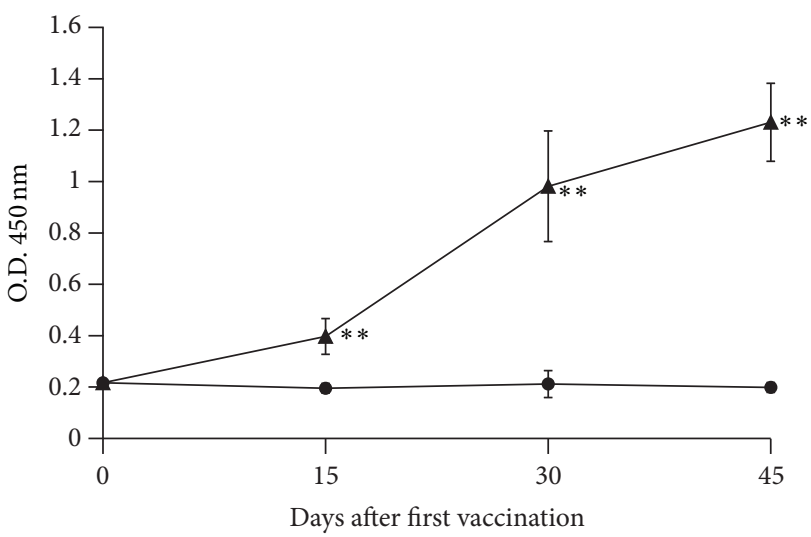

PBS+ISA206

rSjLGL+ISA206

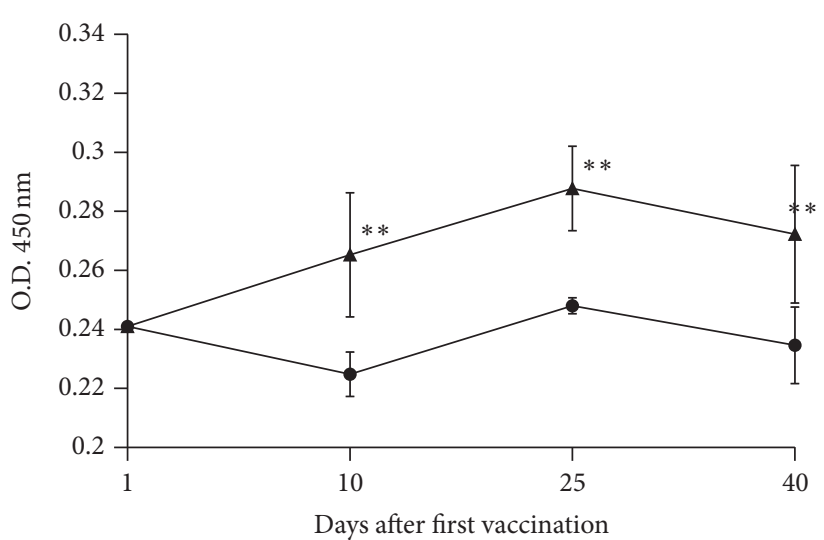

PBS+ISA206

rSjLGL+ISA206

(a)

(b)

FIGURE 3: Kinetics of the induction of specific anti-rSjLGL IgG and IgE antibodies in immunized mice. (a) Sera of 10 immunized mice/group were collected on d $0,15,30$, and 45 after the first immunization and analyzed by ELISA. (b) Sera of 10 immunized mice/group were collected on d $0,10,15$, and 40 after the first immunization and analyzed by ELISA. The results are presented as the mean absorbance at $450 \mathrm{~nm}\left(A_{450}\right)$ of one trial measured for one experiment for each group. Statistically significant differences in the antibody levels of vaccinated mice compared with those of the PBS + adjuvant control group are denoted by ${ }^{* *}(p<0.01)$. 
schistosomiasis [28]. Additionally, in this study, not only did vaccination with SjLGL induce a statistically significant increase in the IgG antibody titer, but also vaccination with SjLGL significantly increased the IgE antibody titer, which is important because the IgE response to adult worm antigens is related to resistance to reinfection $[29,30]$. Together, these results suggest that SjLGL is a candidate vaccine to control schistosomiasis by reducing the liver egg burden and the egg hatching rate.

\section{Competing Interests}

The authors declare that they have no competing interests.

\section{Acknowledgments}

The authors thank Mr. Meifu Tang and Mr. Yaojun Shi at the Shanghai Veterinary Research Institute, Chinese Academy of Agricultural Sciences, for their contribution to the maintenance of parasites and the isolation of the cercariae that were used in this study. They thank International Science Editing for revising the manuscript and their valuable comments. This work was supported by the State Key Laboratory of Veterinary Etiological Biology, Lanzhou Veterinary Research Institute, Chinese Academy of Agricultural Sciences (Grant no. SKLVEB2015KFKT0014), the Chinese National Natural Science Foundation (Grant no. 31672245), and the Natural Science Foundation of Shanghai, China (Grant no. 16ZR1444100).

\section{References}

[1] M. J. Van der Werf, S. J. de Vlas, S. Brooker et al., "Quantification of clinical morbidity associated with schistosome infection in sub-Saharan Africa," Acta Tropica, vol. 86, no. 2-3, pp. 125-139, 2003.

[2] W. C. Campbell, "The chemotherapy of parasitic infections," The Journal of Parasitology, vol. 72, no. 1, pp. 45-61, 1986.

[3] N. R. Bergquist, "Schistosomiasis: from risk assessment to control," Trends in Parasitology, vol. 18, no. 7, pp. 309-314, 2002.

[4] V. R. Bergquist and D. G. Colley, "Schistosomiasis vaccines: research to development," Parasitology Today, vol. 14, no. 3, pp. 99-104, 1998.

[5] T. Yamanaka, Y. Horikoshi, Y. Sugiyama et al., "Mammalian Lgl forms a protein complex with PAR- 6 and aPKC independently of PAR-3 to regulate epithelial cell polarity," Current Biology, vol. 13, no. 9, pp. 734-743, 2003.

[6] A. Müsch, D. Cohen, C. Yeaman, W. J. Nelson, E. RodriguezBoulan, and P. J. Brennwald, "Mammalian homolog of Drosophila tumor suppressor lethal (2) giant larvae interacts with basolateral exocytic machinery in Madin-Darby canine kidney cells," Molecular Biology of the Cell, vol. 13, no. 1, pp. 158168, 2002.

[7] L. M. Kallay, A. McNickle, P. J. Brennwald, A. L. Hubbard, and L. T. Braiterman, "Scribble associates with two polarity proteins, Lgl2 and Vangl2, via distinct molecular domains," Journal of Cellular Biochemistry, vol. 99, no. 2, pp. 647-664, 2006.

[8] G. P. Bell, G. C. Fletcher, R. Brain, and B. J. Thompson, "Aurora kinases phosphorylate Lgl to induce mitotic spindle orientation in drosophila epithelia," Current Biology, vol. 25, no. 1, pp. 61-68, 2015.

[9] J. Zhu, Y. Shang, Q. Wan et al., "Phosphorylation-dependent interaction between tumor suppressors Dlg and Lgl," Cell Research, vol. 24, no. 4, pp. 451-463, 2014.

[10] C. A. Carvalho, S. Moreira, G. Ventura, C. E. Sunkel, and E. Morais-De-Sá, "Aurora a triggers Lgl cortical release during symmetric division to control planar spindle orientation," Current Biology, vol. 25, no. 1, pp. 53-60, 2015.

[11] D. Bilder, "Epithelial polarity and proliferation control: Links from the Drosophila neoplastictumor suppressors," Genes \& Development, vol. 18, no. 16, pp. 1909-1925, 2004.

[12] B. Suresh, S. Ramakrishna, Y.-S. Kim, S.-M. Kim, M.-S. Kim, and K.-H. Baek, "Stability and function of mammalian lethal giant larvae-1 oncoprotein are regulated by the scaffolding protein RanBPM," The Journal of Biological Chemistry, vol. 285, no. 46, pp. 35340-35349, 2010.

[13] T. Ohshiro, T. Yagami, C. Zhang, and F. Matsuzaki, "Role of cortical tumour-suppressor proteins in asymmetric division of Drosophila neuroblast," Nature, vol. 408, no. 6812, pp. 593-596, 2000.

[14] C.-Y. Peng, L. Manning, R. Albertson, and C. Q. Doe, "The tumour-suppressor genes $l g l$ and $d l g$ regulate basal protein targeting in Drosophila neuroblasts," Nature, vol. 408, no. 6812, pp. 596-600, 2000.

[15] C. B. Bridges and K. S. Brehme, The Mutants of Drosophila Melanogaster, Carnegie Institute of Washington, Washington, DC, USA, 1944.

[16] F. Froldi, M. Ziosi, G. Tomba et al., "Drosophila lethal giant larvae neoplastic mutant as a genetic tool for cancer modeling," Current Genomics, vol. 9, no. 3, pp. 147-154, 2008.

[17] K.-H. Baek, Y.-S. Kim, S. Jung, K. Y. Lee, H.-K. Choi, and K.$\mathrm{S}$. Kim, "Molecular cloning and characterization of bovine bgl1 , a novel family member of WD-40 repeat-containing lethal giant larvae tumor suppressor genes," International Journal of Oncology, vol. 20, no. 4, pp. 739-744, 2002.

[18] Y. S. Kim, K. H. Baek, K. Y. Lee et al., "The $r g l-1$ is a legitimate homologue of lethal giant larvae recessive oncogene in rat," International Journal of Oncology, vol. 20, pp. 1219-1225, 2002.

[19] K. Kuwabara, Y. Takahashi, D. Tomotsune, N. Takahashi, and R. Kominami, " $m g l-1$, a mouse homologue of the Drosophila tumor-suppressor gene $l(2) g l$, maps to chromosome 11," Genomics, vol. 20, no. 2, pp. 337-338, 1994.

[20] Y. Cao, Y. Shi, H. Qiao et al., "Distribution of lethal giant larvae ( $\mathrm{Lgl}$ ) protein in the tegument and negative impact of siRNA-based gene silencing on worm surface structure and egg hatching in Schistosoma japonicum," Parasitology Research, vol. 113, no. 1, pp. 1-9, 2014.

[21] F. C. Cardoso, G. C. Macedo, E. Gava et al., "Schistosoma mansoni tegument protein Sm29 is able to induce a Thl-type of immune response and protection against parasite infection," PLoS Neglected Tropical Diseases, vol. 2, no. 10, article e308, 2008.

[22] M. H. Tran, M. S. Pearson, J. M. Bethony et al., “Tetraspanins on the surface of Schistosoma mansoni are protective antigens against schistosomiasis," Nature Medicine, vol. 12, no. 7, pp. 835840, 2006.

[23] P. Humbert, S. Russell, and H. Richardson, "Dlg, scribble and Lgl in cell polarity, cell proliferation and cancer," BioEssays, vol. 25, no. 6, pp. 542-553, 2003. 
[24] B. M. Mechler, W. McGinnis, and W. J. Gehring, "Molecular cloning of lethal giant larvae, a recessive oncogene of Drosophila melanogaster," The EMBO Journal, vol. 4, pp. 1551-1557, 1985.

[25] N. Agrawal, M. Kango, A. Mishra, and P. Sinha, "Neoplastic transformation and aberrant cell-cell interactions in genetic mosaics of lethal(2)giant larvae (lgl), a tumor suppressor gene of Drosophila," Developmental Biology, vol. 172, no. 1, pp. 218229, 1995

[26] S. Lu, "Combination DNA plus protein HIV vaccines," Springer Seminars in Immunopathology, vol. 28, no. 3, pp. 255-265, 2006.

[27] S. L. Zhou, Schistosoma Science, Science Press, Beijing, China, 2nd edition, 2001.

[28] Y. Shi, P. Liu, Y. Yang, J. Liu, and J. Lin, "Cloning and expressing the lethal giant larvae (LGL) gene fragment of Schistosoma japonnicum and valuating the immunoprotective effect of truncated LGL," Chinese Journal of Preventive Veterinary Medicine, vol. 33, pp. 557-561, 2011.

[29] D. W. Dunne, A. E. Butterworth, A. J. C. Fulford et al., "Immunity after treatment of human schistosomiasis: association between $\operatorname{IgE}$ antibodies to adult worm antigens and resistance to reinfection," European Journal of Immunology, vol. 22, no. 6, pp. 1483-1494, 1992.

[30] P. Hagan, U. J. Blumenthalt, D. Dunn, A. J. G. Simpson, and H. A. Wilkins, "Human IgE, IgG4 and resistance to reinfection with Schistosoma haematobium," Nature, vol. 349, no. 6306, pp. 243-245, 1991. 

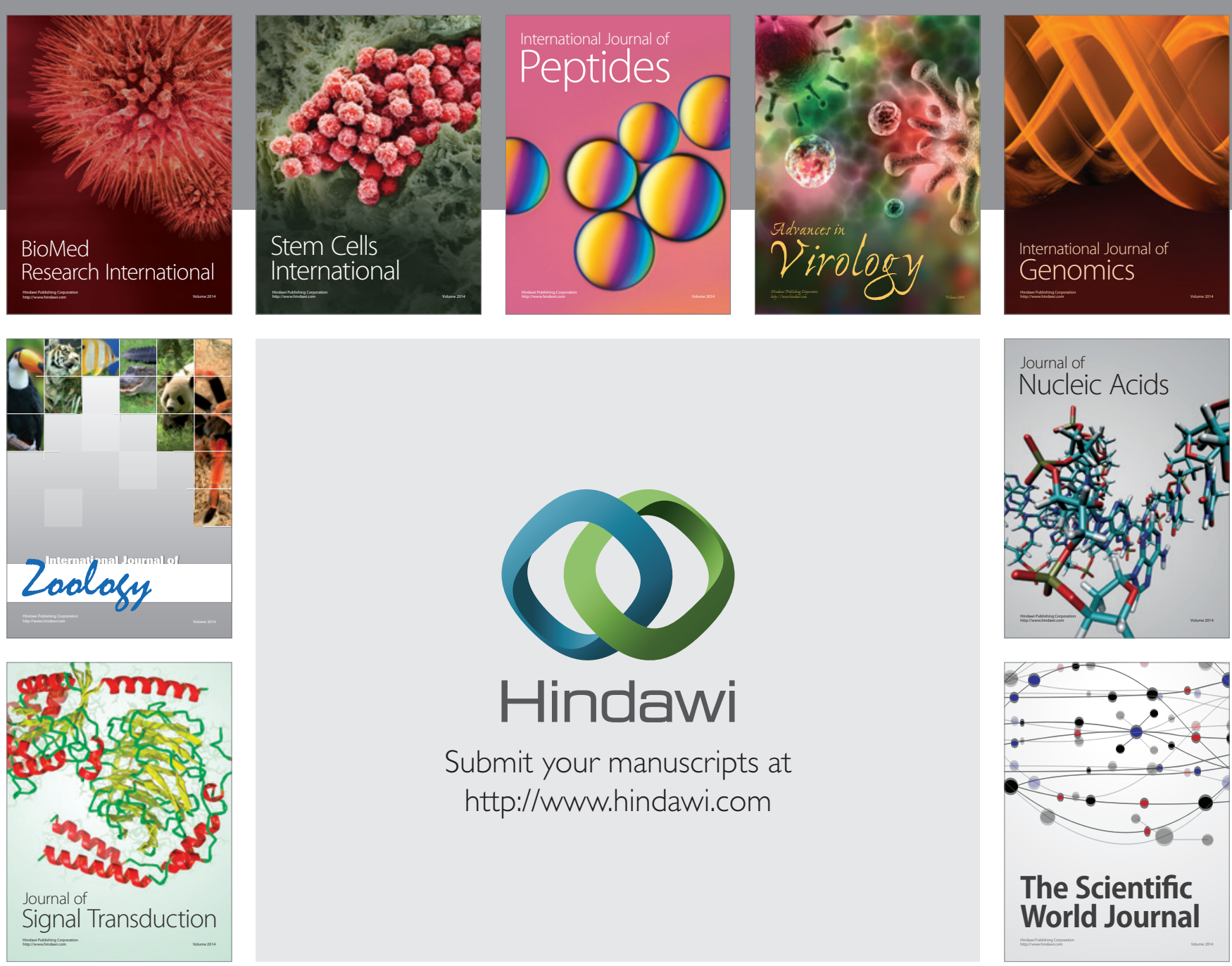

Submit your manuscripts at

http://www.hindawi.com
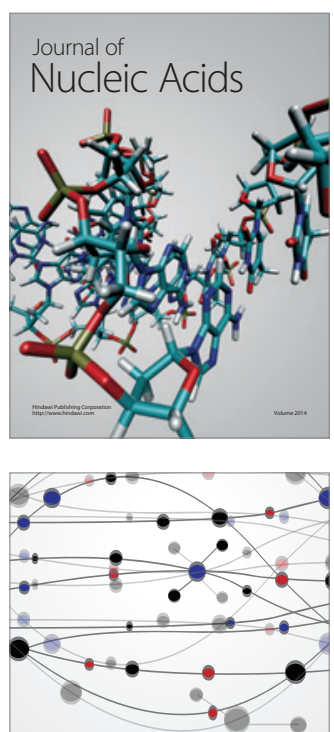

The Scientific World Journal
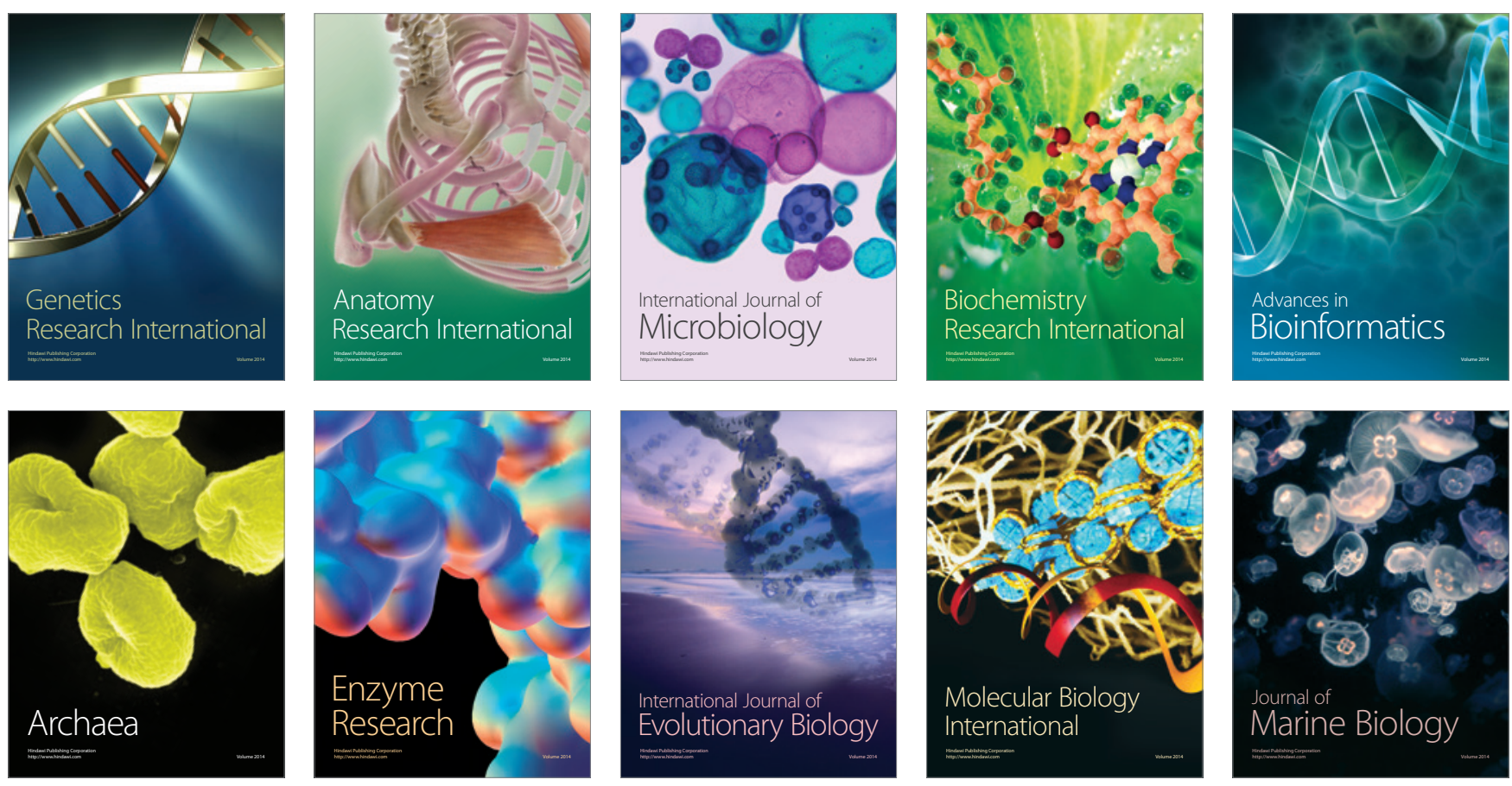